\title{
Carmen Sippl
}

Pädagogische Hochschule Niederösterreich, Campus Baden

\section{Wertstoff}

DOI: https://doi.org/10.53349/sv.2021.i2.a81

Wertstoff, der. Substantiv, maskulin.

Der Duden definiert ,Wertstoff' als „im Abfall, Müll enthaltener Altstoff, der als Rohstoff erneut verwendet werden kann" (Duden online). Dass hier dem Weggeworfenen, Entsorgten ein Wert zugeschrieben wird, erscheint zunächst paradox: Warum sollte man etwas Wertvolles wegwerfen, ein Ding, dem eine solch besondere Qualität (ein Wert) innewohnt, sodass es jemandem bedeutsam ist?

Altstoff als Rohstoff zu betrachten, der wertvoll ist, richtet den Blick einerseits auf die Endlichkeit natürlicher Ressourcen. Fossile Energieträger wie „Kohle, Erdöl und Erdgas, die aus vor Jahrmillionen abgestorbenenen Tieren und Pflanzen unter Ausschluss von Luftsauerstoff entstanden sind" (Zea-Schmidt \& Hamann 2013, 34), verwenden wir so intensiv als Brennstoffe, als würden wir an deren Nachwachsen wie Sonnenblumen auf dem Feld im nächsten Sommer glauben.

Zum anderen rückt daher ein Verständnis für das Funktionieren des Erdsystems als ökologischer Kreislauf in den Mittelpunkt: Altstoff wird zum Rohstoff und damit zum Wertstoff.

Muss z.B. ein Haus wegen Altersschwäche abgerissen werden, werden die abgebauten, zerlegten Materialien in einem Lager gesammelt (vgl. Urban Mining) und dann, als wertvolle Ressourcen, weiterverwendet. Um insbesondere die Unmengen an Plastik, welche täglich in Haushalten und Industrie als Abfall anfallen, zu recyceln - also in einen Kreislauf der Wiederverwertung zu geben - bedarf es kreativer Lösungen, von der Vereinfachung der Sammelsysteme bis zu innovativen Produktionsweisen und Produktdesigns. Als Vorbild kann die „Biosphäre als Modell für die Technosphäre im Anthropozän" (Leinfelder 2020) dienen.

Meistens belehre erst der Verlust uns über den Wert der Dinge, bemerkte Arthur Schopenhauer. Damit es nicht so weit kommt, bedarf es also vor allem kreativer, innovativer Prozesse, um anstelle von Abfall Wertstoffe zu produzieren, mit erneuerbaren Energien. „Müll fiele 


\section{\# schuleverantworten}

führungskultur_innovation_autonomie

in einem solchen System nicht an", schreibt der Geologe und Anthropozän-Forscher Reinhold Leinfelder. Wer würde auch etwas Wertvolles entsorgen wollen?

\section{Literaturverzeichnis}

Duden online, „Wertstoff“. URL: https://www.duden.de/rechtschreibung/Wertstoff

Leinfelder, Reinhold (21. Oktober 2020). „Auch Maschinen haben Hunger“ - Die Biosphäre als Modell für die Technosphäre im Anthropozän. SciLog - Der Anthropozäniker. URL: https://scilogs.spektrum.de/der-anthropozaeniker/auch-maschinen-haben-hunger/

Urban Mining - Energie- und Ressourceneinsparung durch Urban Mining-Ansätze. Ein Projekt der TU Wien. URL: https://nachhaltigwirtschaften.at/de/sdz/projekte/urban-mining.php

Zea-Schmidt, Claudia \& Hamann, Alexandra (2013). Lernen in globalen Zusammenhängen. Die große Transformation. Jahrgangsstufen 9 und 10. Materialien für den Unterricht. Berlin. URL: http://www.berlin.de/sen/bildung/unterricht/faecher-rahmenlehrplaene/globale-entwicklung/die_grosse_transformation_web.pdf

\section{Tipp}

Reinhold Leinfelders informativen und inspirierenden Vortrag zum Thema „,Auch Maschinen haben Hunger' - Die Biosphäre als Modell für die Technosphäre im Anthropozän“ vom 23. April 2021 (im Rahmen des Symposiums „Kulturelle Nachhaltigkeit lernen und lehren“ der Pädagogischen Hochschule Niederösterreich) gibt es hier zum Nachschauen: https://www.ph-noe.ac.at/de/forschung/forschungund-entwicklung/anthropozaen/symposium

\section{Autorin}

Carmen Sippl, HS-Prof. Mag. Dr.

Hochschulprofessorin für Kultursemiotik und Mehrsprachigkeit an der Pädagogischen Hochschule Niederösterreich und Lehrbeauftragte an der Philologisch-Kulturwissenschaftlichen Fakultät der Universität Wien. Im Projekt „Das Anthropozän lernen und lehren“ (http://anthropozaen.ph-noe.ac.at/) beschäftigt sich die Philologin mit der Rolle der kulturellen Bildung für die Neugestaltung der Mensch-Natur-Beziehung im Anthropozän.

Kontakt: carmen.sippl@ph-noe.ac.at 